\title{
A Non-steady State Model for the Austenite-to-ferrite Transformation Kinetics under the Non-partition Condition in Fe-C-Mn Alloys
}

\author{
Akira SEKI, ${ }^{1) *}$ Kotarou HAYASHI, ${ }^{2)}$ Takafumi AMINO, ${ }^{2)}$ Genichi SHIGESATO, ${ }^{3)}$ Takayuki NOZAKI, ${ }^{4)}$ \\ Masafumi AZUMA ${ }^{4)}$ and Masayoshi SUEHIRO' \\ 1) Nippon Steel Technology Co., Ltd., 1-8 Fuso-Cho, Amagasaki, Hyogo, 660-0891 Japan. \\ 2) Nippon Steel Corporation, 1-8 Fuso-Cho, Amagasaki, Hyogo, 660-0891 Japan. \\ 3) Nippon Steel Corporation, 20-1 Shintomi, Futtsu, Ciba, 293-8511 Japan. \\ 4) Nippon Steel Corporation, 5-3 Tokaimachi, Tokai, Aichi, 476-8686 Japan. \\ 5) Nippon Steel Technology Co., Ltd., 1-7-1 Yurakucho, Chiyoda-ku, Tokyo, 100-0006 Japan.
}

(Received on April 3, 2019; accepted on June 25, 2019)

\begin{abstract}
A non-steady state model describing the transition from the para-equilibrium to the local equilibrium under the non- or negligible-partition condition is proposed to investigate the austenite to ferrite transformation kinetics in Fe-C-Mn steels. The present model has been developed based on the quasi-steady state model used for calculating the solute drag force by the segregated solute within the ferrite/austenite interface, although the solute-drag force is not calculated in the present model. The calculated Mn profiles are compared with the STEM-EELS observation reported previously by some of the present authors. The temporal evolution of the Mn profiles during PE to NPLE is shown. Combining a simple C diffusion model, the transition path from PE to NPLE on the C-Mn isothermal section of an Fe-C-Mn alloy is also presented.
\end{abstract}

KEY WORDS: austenite to ferrite transformation; interface migration; non-steady state model; kinetics model; para-equilibrium; local equilibrium; non-partition; negligible partition.

\section{Introduction}

In the diffusion controlled growth of the ferrite $(\alpha)$ grains from the austenite $(\gamma)$ parent phase in $\mathrm{Fe}-\mathrm{C}-\mathrm{X}$ ternary alloys, where $\mathrm{X}$ is a slow diffusing substitutional alloying element, there are two $\alpha$-growth modes: ${ }^{1,2)}$ one, where the slow diffusing element $\mathrm{X}$ is partitioned between $\alpha$ - and $\gamma$ - phase; a second, where $\mathrm{X}$ element cannot be partitioned between these two phases. The latter partitionless growth mode is called as non-partition or negligible-partition (NP) transformation mode. In the NP transformation mode, the rate of the transformation is controlled by the diffusion of carbon (C) while X element is considered immobile. Under the NP transformation, two extreme models have been proposed further. The first one is the para-equilibrium (PE) model, ${ }^{1-3)}$ where no distribution of $\mathrm{X}$ in the $\alpha / \gamma$ interface, and the second one is the non-partition or negligible-partition local equilibrium model (NPLE), ${ }^{1,2)}$ where the full local equilibrium is established resulting in spike of $\mathrm{X}$ element in front of the $\alpha / \gamma$ interface. Hillert ${ }^{2)}$ discussed the transition from PE to PLE (full local equilibrium under partition condition) through the NPLE. In the process from NPLE to PLE, the partitioning of $\mathrm{X}$ between $\alpha$ and $\gamma$ occurs and the

* Corresponding author: E-mail: seki.akira.s5m@nstec.nipponsteel.com DOI: https://doi.org/10.2355/isijinternational.ISIJINT-2019-216
$\mathrm{C}$ and $\mathrm{X}$ concentrations change along $\alpha / \gamma$ full equilibrium boundary in the isothermal section of phase diagram.

Odqvist et $a l .{ }^{4)}$ proposed a model describing a continuous change from PE to NPLE during partitionless growth of $\alpha$ in an $\mathrm{Fe}-\mathrm{C}-\mathrm{Ni}$ alloy in the isothermal condition. They used the quasi-steady state solute drag model ${ }^{5)}$ and calculated the Ni profile by balancing the dissipation of Gibbs energy and the driving force for the fixed interface migration velocity to obtain the PE to NPLE trajectory on the isothermal section of $\mathrm{Ni}-\mathrm{C}$ phase diagram. It was assumed that $\mathrm{C}$ controls the rate of the transformation.

The purpose of the present study is to develop a new model describing the temporal transition from PE to NPLE continuously during $\gamma$ to $\alpha$ isothermal transformations. The developed model is a non-steady state (time-dependent) one, which is an extension of the quasi-steady state model used for describing the solute drag effect in the $\gamma$ to $\alpha$ transformation of $\mathrm{Fe}-\mathrm{C}-\mathrm{X}$ alloys by many authors. ${ }^{5-12)} \mathrm{A}$ non-steady state (time-dependent) solute drag model was proposed by Murakami et al. ${ }^{13)}$ to calculate the development of interface segregation during ferrite transformation. In their model the austenite/ferrite interface moving velocity is calculated from the growth rate of ferrite. In the present model, the velocity of the moving interface is included explicitly in the basic equation.

To describe the deviation from local equilibrium at the 
moving phase interface during the transformation, the interaction between an alloying element and the interface must be considered. Therefore the present model is based on the solute drag model, although the solute drag force ${ }^{14)}$ and the dissipation energy ${ }^{15}$ are not calculated in the present model. The calculations using the solute drag force and the dissipation energy often lead to very different conclusions if they are not properly treated. ${ }^{16)}$ In the present model, the temporal evolution of $\mathrm{X}$ element profiles can be calculated. Hereafter, $\mathrm{X}$ is considered as $\mathrm{Mn}$. In steels containing a certain amount of $\mathrm{Mn}$, the micro-structure is usually controlled to be a $\alpha / \gamma$ dual phase structure by heat treatment in order to concentrate $\mathrm{Mn}$ and $\mathrm{C}$ atoms into the austenite phase in order to stabilize the austenite. ${ }^{17,18)}$

The analysis by the present model will be focused on the experimental results previously reported by the some of the present authors. ${ }^{19)}$ In that paper, the Mn concentration profiles around the transformation interfaces during the growth of allotriomorphic ferrite under isothermal annealing at $700^{\circ} \mathrm{C}$ in $\mathrm{Fe}-0.12 \mathrm{C}-2.0 \mathrm{Mn}$ alloy (in wt.\%) are presented using the aberration corrected STEM-EELS technique. ${ }^{20)}$ The transformation process reported there can be considered to be the transition from PE to NPLE. In the present study the temporal evolution of the Mn profiles are computed using the non-steady state kinetic model to reveal the transition process from PE to full LE under the NP condition. The comparison between the experimental ${ }^{19)}$ and the calculated results will be made to show that the present model can describe the austenite to ferrite transformation well.

\section{Model}

During diffusional phase transformations, solute elements such as $\mathrm{C}$ and $\mathrm{Mn}$, diffuse across the phase boundary to the other phase. In the case of $\gamma$ to $\alpha$ transformation, the austenite stabilizer, $\mathrm{C}$ and $\mathrm{Mn}$ atoms diffuse to $\alpha$ phase from $\gamma$ phase. The present model, which describes diffusional phase transformation, is based on the one-dimensional diffusion equation for one solute element.

\subsection{Equation in a Rest Frame}

The flux conservation equation is expressed by the following.

$$
\frac{\partial c}{\partial t}=-\frac{\partial}{\partial x} J
$$

where $c$ is the solute concentration, $t$ is time, $x$ is distance, $J$ is the solute flux. The flux $J$ is given by the gradient of the chemical potential $\mu$.

$$
J=-c M \frac{\partial \mu}{\partial x}
$$

where $M$ is a diffusion mobility and it is related to the diffusion coefficient $D$ by Einstein's relation below.

$$
D=M R T
$$

The $\mu$ is given using the solute activity $a$ as the following. ${ }^{21)}$

$$
\mu=\mu_{0}+R T \ln a
$$

where $\mu_{0}$ is a constant, $R$ is gas constant, $T$ is temperature in
Kelvin. The following relation between $a$ and $c$ holds using the activity coefficient $f$.

$$
a=f \cdot c
$$

Coupling Eqs. (1)-(4), the following equation containing the solute concentration $c$ and the solute activity $a$ is obtained.

$$
\frac{\partial c}{\partial t}=\frac{\partial}{\partial x}\left(c D \frac{1}{a} \frac{\partial a}{\partial x}\right)
$$

Using Eq. (5), the equation for activity $a$ is obtained from Eq. (6).

$$
\frac{\partial}{\partial t}\left(\frac{a}{f}\right)=\frac{\partial}{\partial x}\left(\frac{D}{f} \frac{\partial a}{\partial x}\right)
$$

\subsection{Equation in a Moving Frame}

In the case that a boundary moves with velocity $v$, such as the phase transformation, Eq. (7) in a rest frame $(x, t)$ should be converted to the equation in a moving frame $\left(x^{\prime}, t^{\prime}\right)$ with velocity $v$. The coordinate transformation relation between $(x, t)$ and $\left(x^{\prime}, t^{\prime}\right)$ is following.

$$
x^{\prime}=x-\int_{0}^{t} v(\tau) d \tau, t^{\prime}=t
$$

where $v$ may be a function of time. The derivatives, $\partial / \partial x$ and $\partial / \partial t$ are expressed by $\partial / \partial x^{\prime}$ and $\partial / \partial t^{\prime}$ using the chain rule of derivatives as follows.

$$
\begin{aligned}
& \frac{\partial}{\partial x}=\frac{\partial x^{\prime}}{\partial x} \frac{\partial}{\partial x^{\prime}}+\frac{\partial t^{\prime}}{\partial x} \frac{\partial}{\partial t^{\prime}}=\frac{\partial}{\partial x^{\prime}} \\
& \frac{\partial}{\partial t}=\frac{\partial x^{\prime}}{\partial t} \frac{\partial}{\partial x^{\prime}}+\frac{\partial t^{\prime}}{\partial t} \frac{\partial}{\partial t^{\prime}}=-v(\tau) \frac{\partial}{\partial x^{\prime}}+\frac{\partial}{\partial t^{\prime}}
\end{aligned}
$$

Using Eq. (9), Eq. (7) is transformed to the following equation.

$$
\frac{\partial}{\partial t^{\prime}}\left(\frac{a}{f}\right)=\frac{\partial}{\partial x^{\prime}}\left(v \frac{a}{f}+\frac{D}{f} \frac{\partial a}{\partial x^{\prime}}\right)
$$

Superscripts in $x^{\prime}$ and $t^{\prime}$ are removed as it is a matter of notation. Then the equation in a moving frame is obtained as follows.

$$
\frac{\partial}{\partial t}\left(\frac{a}{f}\right)=\frac{\partial}{\partial x}\left(v \frac{a}{f}+\frac{D}{f} \frac{\partial a}{\partial x}\right)
$$

Here, $D$ and $f$ are functions of $x$ and $t$, generally. Using Eq. (5), the following equation for the solute concentration $c$ is obtained.

$$
\frac{\partial c}{\partial t}=\frac{\partial}{\partial x}\left(v c+\frac{D}{R T} \frac{\partial E}{\partial x} c+D \frac{\partial c}{\partial x}\right)
$$

Here,

$$
E \equiv R T \ln f
$$

where $E$ is the interaction potential of the solute with the interface.

In Eq. (11) the coordinate derivative of $E$ appears. Thus, it is easier to solve Eq. (10) numerically than Eq. (11). In this paper, Eq. (10) is used as the basic equation to be solved. Equation (10) is a time-dependent partial differential equation for the activity $a(x, t) . a(x, t)$ is obtained by solving Eq. (10) numerically using the implicit method, ${ }^{22,23)}$ then 
the concentration, $c(x, t)$ is calculated from $a(x, t)$ and $f(x)$ using Eq. (5).

\subsection{Activity Coefficient Distribution}

In the present model, during diffusional phase transformations, solutes segregate favorably into the potential well inside the interface region. The interface to be considered in this paper has the width, thus the term "interface region" is used. In the literatures, ${ }^{5-11)}$ a triangular interaction potential well in the interface region is assumed. In the present model, triangular potential wells are adopted too. The potential well is transformed to the activity coefficient distribution using Eq. (12). Figure 1(a) is an example of such interaction potential, which is for $\gamma$ stabilizer. In the figure, the center of the interface region $(\varphi)$ is set to be the origin of $x$-cordinate. The $x$-cordinates of the boundaries of $\alpha / \varphi$ and the $\varphi / \gamma$ are defined as $-\delta$ and $\delta$, respectively. That is, the width of the interface is $2 \delta$. The potential values, $E_{\alpha}$ and $E_{\gamma}$, are defined for ferrite and austenite, respectively. The potential value $E_{g b}$ is defined at the center of the interface region, which corresponds to a cusp in a triangular potential curve. The activity coefficient $f_{\alpha}, f_{g b}$ and $f_{\gamma}$ correspond to the potential $E_{\alpha}, E_{g b}$ and $E_{\gamma}$, respectively. The potential of the $\alpha$ phase is set to zero as a reference. Then the value of $f_{\alpha}$ is one using Eq. (12). The effective potential depth $E_{0}$ and half of the potential difference between $\alpha$ and $\gamma$ phase $\Delta E$ are introduced. They are defined in Fig. 1(a) and are positive. $\Delta E$ is related to the solute partition coefficient $k^{\gamma / \alpha}$ by the following equation.

$$
2 \Delta E=R T \ln k^{\gamma / \alpha}
$$

(a)

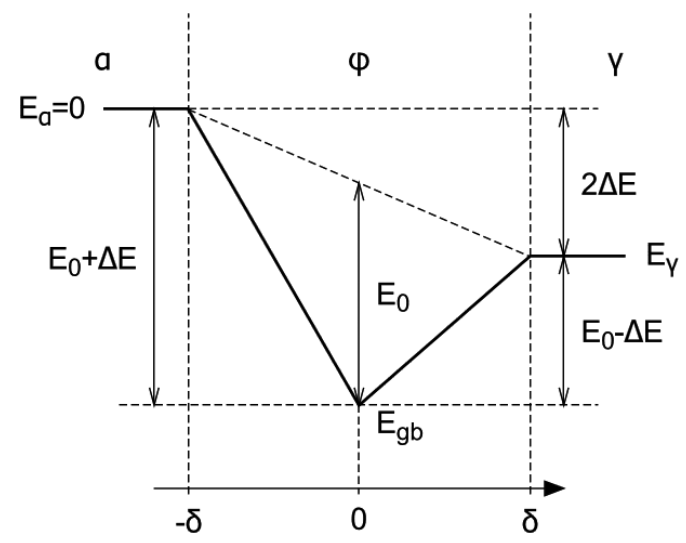

(b)

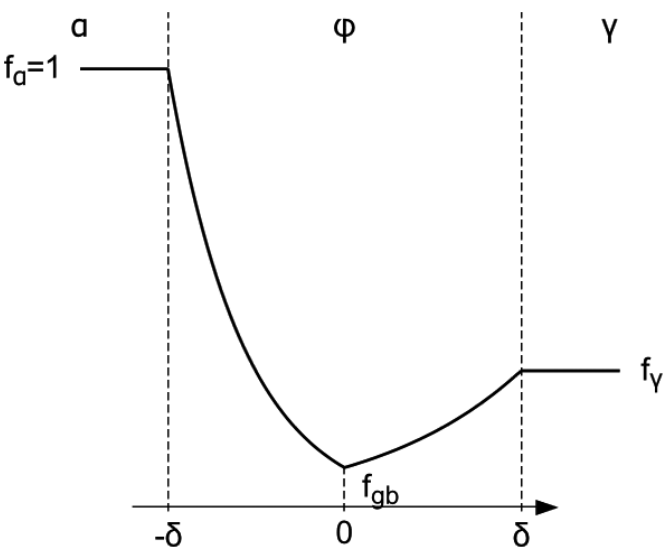

Fig. 1. Illustrations of (a) a potential well and (b) a corresponding activity coefficient profile for the $\gamma$ stabilizer, $\mathrm{Mn}$.
Using $E_{0}$ and $\Delta E$, the potentials, $E_{g b}$ and $E_{\gamma}$ are expressed as follows.

$$
\begin{gathered}
E_{g b}=-E_{0}-\Delta E=R T \ln f_{g b} \\
E_{\gamma}=-2 \Delta E=R T \ln f_{\gamma} . .
\end{gathered}
$$

Figure 1(b) shows the activity coefficient distribution corresponding to the potential in Fig. 1(a). The curve is not triangular obviously.

It should be stressed that the present model does not include the interface migration mechanism in it and just calculates the temporal evolution of the solute distribution inside and around the interface under the given interface migration velocity.

\section{Experimental Results ${ }^{19)}$}

$\mathrm{Fe}-0.12 \mathrm{C}-2 \mathrm{Mn}$ (all in wt.\%) alloy was annealed at $700^{\circ} \mathrm{C}$ for 30, 300, 3000 and $10000 \mathrm{~s}$. The concentration at the ferrite/MA interfaces, which were the $\alpha / \gamma$ interfaces at $700^{\circ} \mathrm{C}$, were measured using aberration corrected STEM-EELS. ${ }^{20}$ ) The corrected concentration profiles, which should agree with the true profiles, were obtained from the postulated true profiles by the convolution with the broadening factors. The true Mn concentration profiles are characterized by the peak concentration at the interface, the concentration at the $\alpha / \varphi$ boundary, the concentration at the $\varphi / \gamma(\mathrm{MA})$ boundary and the width of the $\mathrm{Mn}$ enrichment region in the austenite. They will be compared with the profiles calculated using the present non-steady state model. Figure 2 shows the temporal change of the thickness of ferrite (size of the ferrite grains) obtained by optical microscope observation, which is necessary to perform the simulations. The curve in the figure is an approximate third-order polynomial function of square root of time.

\section{Simulation Conditions}

Figure 3 shows the Fe-rich side of the isothermal section of $\mathrm{Fe}-\mathrm{C}-\mathrm{Mn}$ ternary phase diagram at $700^{\circ} \mathrm{C}$ calculated by Thermo-Calc $2018 \mathrm{a}$ and database TCFE8. ${ }^{24)}$ The solid

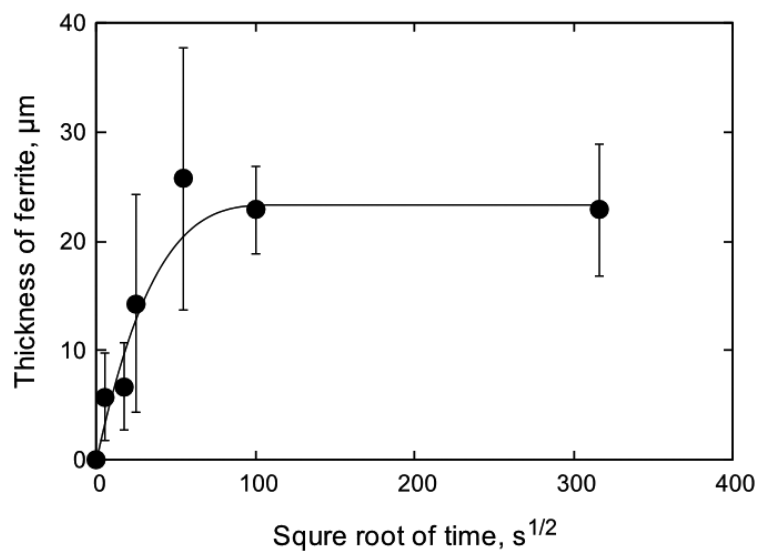

Fig. 2. Temporal change of the thickness of $\alpha$ grain at $700^{\circ} \mathrm{C}$. The solid circles with error bars are values obtained by optical microscope observation. The solid line is the curve approximated by a third order polynomials of square root of time. 


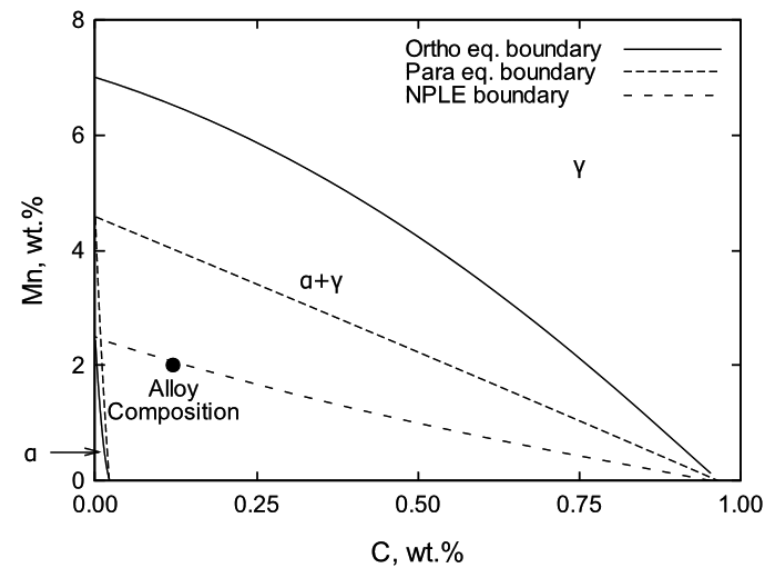

Fig. 3. Fe-rich side of $\mathrm{C}-\mathrm{Mn}$ isothermal section of $\mathrm{Fe}-\mathrm{C}-\mathrm{Mn}$ phase diagram with ortho- equilibrium, PE and NPLE boundaries at $700^{\circ} \mathrm{C}$. Solid circle represents the alloy composition.

circle in the figure is the alloy composition of Fe- $0.12 \mathrm{C}$ $2 \mathrm{Mn}$. The alloy is in the NPLE region. The simulations for Mn are performed solving Eq. (9). The starting state of the simulation is assumed to be PE. The simulations are performed during the transformation from the para-equilibrium to full local equilibrium under non-partition or negligible partition condition. This assumption is reasonable, because the experimental results show that the Mn concentration at the $\alpha / \varphi$ boundary maintains the value of 2 wt. $\%$ during the process. The width of interface $2 \delta$ is assumed to be $1 \mathrm{~nm}$. The diffusion coefficient of $\mathrm{Mn}$ inside the interface $D_{M n}^{i n t}$ is assumed to be constant through the interface region and to be the geometric average of the diffusion coefficients of Mn in ferrite $D_{M n}^{\alpha}$ and in austenite $D_{M n}^{\gamma}$. The values of $D_{M n}^{\alpha}$ and $D_{M n}^{\gamma}$ are calculated using DICTRA 2018a, database TCFE 8 and MOBFE3. ${ }^{24)}$ The values are listed in Table 1. As the simulations are performed under the non-partition or negligible partition (NP) condition, the Mn concentration at the $\alpha / \varphi$ boundary is fixed at the value of the alloy bulk composition ( $2 \mathrm{wt} . \%$ ). The size of the austenite region is set to $2 \mu \mathrm{m}$. This size is enough to eliminate the effect of the end of calculation region and the Mn concentration at the end of austenite is fixed at the bulk composition of the alloy (2 wt.\%). In the fixed concentration boundary conditions, the conservation low of the concentration does not hold. In the present simulations, the Mn concentration at the $\alpha / \varphi$ boundary is fixed in order to fulfill the non-partition condition. Therefore, there is a possibility that the flux of $\mathrm{Mn}$ occurs. This is a consequence of using the finite size model in the simulations.

The effective potential depth $E_{0}$ is treated as a fitting parameter for the experimental results. The value of $\Delta E$ is calculated from $k_{M n}^{\gamma / \alpha}$ using Eq. (13). The value of $k^{\gamma / \alpha}$ is calculated for $0.12 \mathrm{C}-2 \mathrm{Mn}-\mathrm{Fe}$ alloy at $700^{\circ} \mathrm{C}$ using ThermoCalc 2018a and the database TCFE8. ${ }^{24)}$ The calculated value of $k^{\gamma / \alpha}$ is 3.14 , then $\Delta E$ is $4.63 \mathrm{~kJ} / \mathrm{mol}$ using Eq. (13).

It is assumed that the diffusion of $\mathrm{C}$ controls the transformation during the PE to NPLE transition. The diffusion of $\mathrm{C}$ is not considered in the present simulation, thus the interface migration distance or velocity cannot be determined by the model. In the preset study, interface migration velocity is calculated using the experimental time versus ferrite thick-
Table 1. Diffusion coefficients of the ferrite, austenite and interface in $\mathrm{m}^{2} / \mathrm{s}$ at $700^{\circ} \mathrm{C}$.

\begin{tabular}{ccc}
\hline ferrite & austenite & interface \\
\hline $2.48 \cdot 10^{-17}$ & $1.40 \cdot 10^{-19}$ & $1.86 \cdot 10^{-18}$ \\
\hline
\end{tabular}

ness relation (Fig. 2). This relation is approximated by a third-order polynomial of square root of time. The migration velocity is derived by time derivative of this polynomial.

\section{Simulation Results}

The simulations of isothermal austenite to ferrite transformation at $700^{\circ} \mathrm{C}$ were performed for $0.12 \mathrm{C}-2 \mathrm{Mn}-\mathrm{Fe}$ alloy solving Eq. (10) for Mn numerically. The initial configuration of the solutes is the para-equilibrium by only $\mathrm{C}$ diffusion and $\mathrm{Mn}$ distribution is uniform initially through the entire system, which consists of the ferrite, the interface and the austenite regions. Figure 4 shows the temporal changes of (a) the migration distance and (b) velocity calculated from the time versus experimental ferrite thickness. The effective potential in the interface region is treated as a fitting parameter for the experimental results. Figure 5 shows the temporal change of Mn concentration (at.\%) at the center of the interface region $c_{M n}^{\varphi 0}$ from $0 \mathrm{~s}$ to $10^{4} \mathrm{~s}$ for three values of the effective potential $E_{0}$ with the experimental concentrations. The figure shows that the best fitting of $c_{M n}^{\varphi 0}$ with the experiment is obtained for the value of $14.0 \mathrm{~kJ} / \mathrm{mol}$, although the deviation of the experimental and simulated values at $30 \mathrm{~s}$ is significant. The deviation at $30 \mathrm{~s}$ will be discussed later. The values of effective potential depth ranging from 7 to $15 \mathrm{~kJ} / \mathrm{mol}$ for $\mathrm{Mn}$ are reported. ${ }^{5-9)}$ The value of $14.0 \mathrm{~kJ} / \mathrm{mol}$ is within the range of these values, then this value for $E_{0}$ is used in the following simulations.

Figure 6 shows the temporal evolution of $\mathrm{Mn}$ profile at the interface region $(\varphi)$ and the adjacent $\gamma$ region from $0 \mathrm{~s}$ to $10^{4} \mathrm{~s}$. It is shown that the concentration profile at each time has a distorted triangular shape corresponding to the triangular potential shape. The peak concentration of Mn at the center of the interface region $\gamma$ grows with time. At early stage of the transformation $(\sim 300 \mathrm{~s})$, the depletion zone of Mn can be seen near the $\alpha$ side in the interface region $\varphi$. It seems that Mn diffuses from the $\alpha$ region to the $\varphi$ region rapidly at this stage. In the quasi-steady state solute drag model, such depletion zone cannot be seen. ${ }^{5,8,9)}$

Figure 7 shows temporal change of the Mn concentrations at the center of the $\varphi, c_{M n}^{\varphi 0}$ and those at $\varphi / \gamma$ boundary, $c_{M n}^{\varphi / \gamma}$ with the experimental values. $c_{M n}^{\varphi / \gamma}$ is considered to be the Mn spike. The agreement between the calculated and the experimental values for $c_{M n}^{\varphi / \gamma}$ is good. The concentration $c_{M n}^{\varphi 0}$ increases at early stage $\left(\lesssim 10^{-6} \mathrm{~s}\right)$ and remains 6.4 at. $\%$ until $\sim 300 \mathrm{~s}$, then increase to the stationary value with time. On the other hand, $c_{M n}^{\varphi / \gamma}$ remains 2.03 at.\% (2 wt.\%) until $\sim 300 \mathrm{~s}$, then increases to stationary values according to the increase of $c_{M n}^{\varphi 0}$. The ratio of the stationary value of $c_{M n}^{\varphi / \gamma}$ to the value of $c_{M n}^{\alpha / \varphi}(2.03$ at. $\%)$ is 3.14. This value is exactly the equilibrium $\mathrm{Mn}$ partition coefficient at $700^{\circ} \mathrm{C}$. This means that full local equilibrium of $\mathrm{Mn}$ is achieved between $\alpha$ and $\gamma$ across $\varphi$.

Figure 8 shows the Mn concentration profiles across the 
(a)

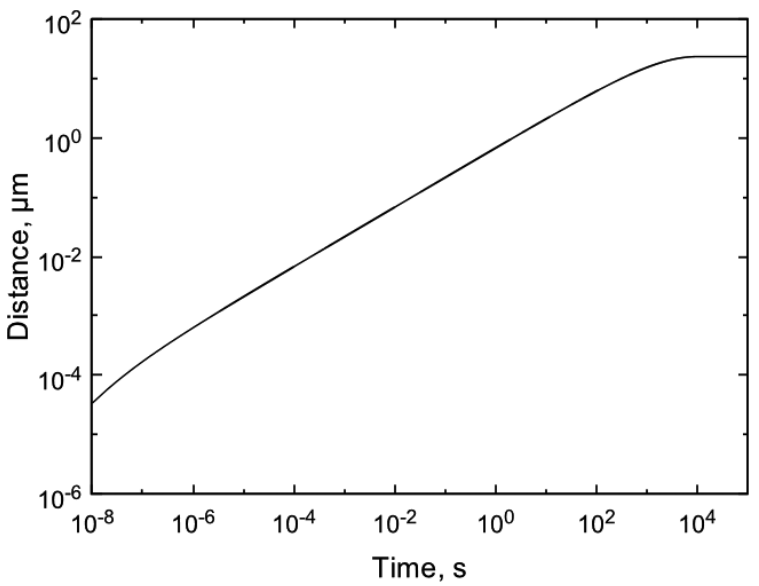

(b)

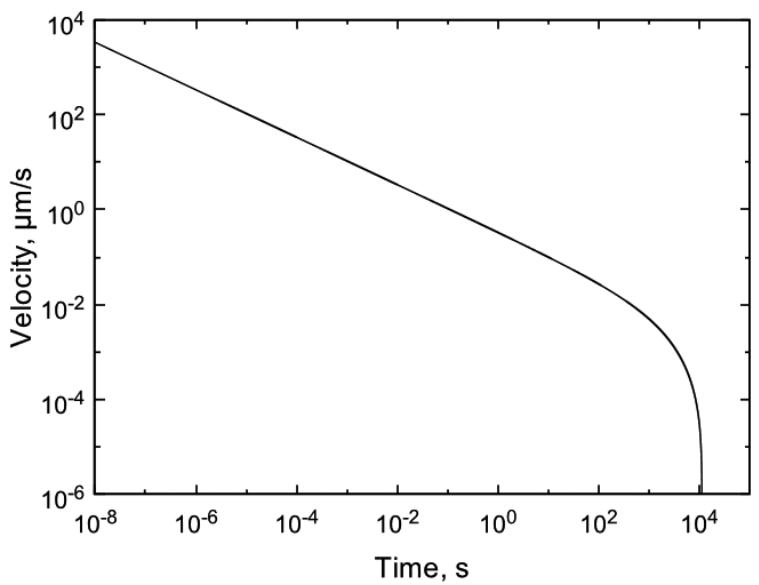

Fig. 4. (a) Distance and (b) Velocity of the interface migration as a function of time.

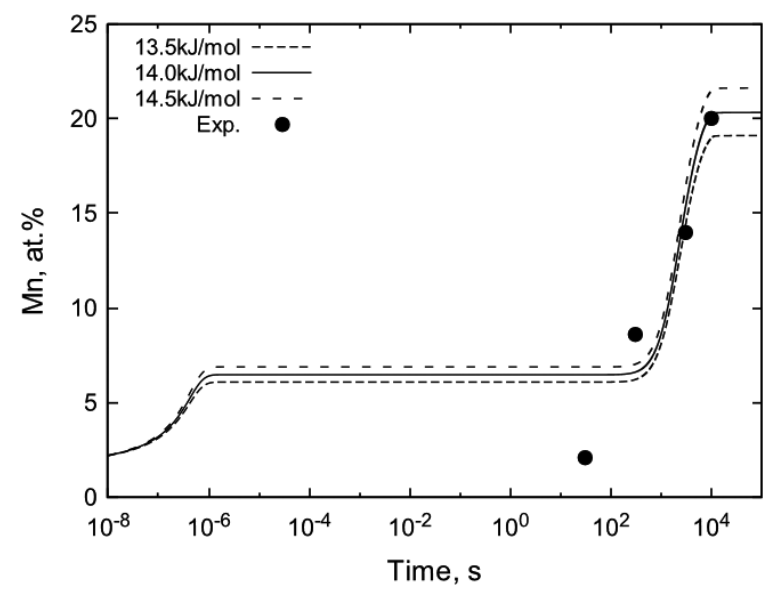

Fig. 5. Mn concentrations at the center of the interface region $\varphi$, $c_{M n}^{\varphi 0}$ as a function of time for three values of effective potential, $E_{0}$. The solid circles show the experimental values obtained by STEM-EELS observations.

interface region, $\varphi$ for the simulations and experiments at (a) $30 \mathrm{~s}$, (b) $300 \mathrm{~s}$, (c) $3000 \mathrm{~s}$, (d) $10000 \mathrm{~s}$.

At $30 \mathrm{~s}$, the experimental profile is flat and it indicates that the para-equilibrium is still maintained. On the other hand $\mathrm{Mn}$ distribution is formed in the simulation. The Mn depletion zone in the half of the interface region, $\varphi$ adjacent to the ferrite and the peak of the Mn concentration in the interface region are observed in the simulated profile. This discrepancy can be explained as follows. In the simulation the incubation time of ferrite nucleation is ignored. It is

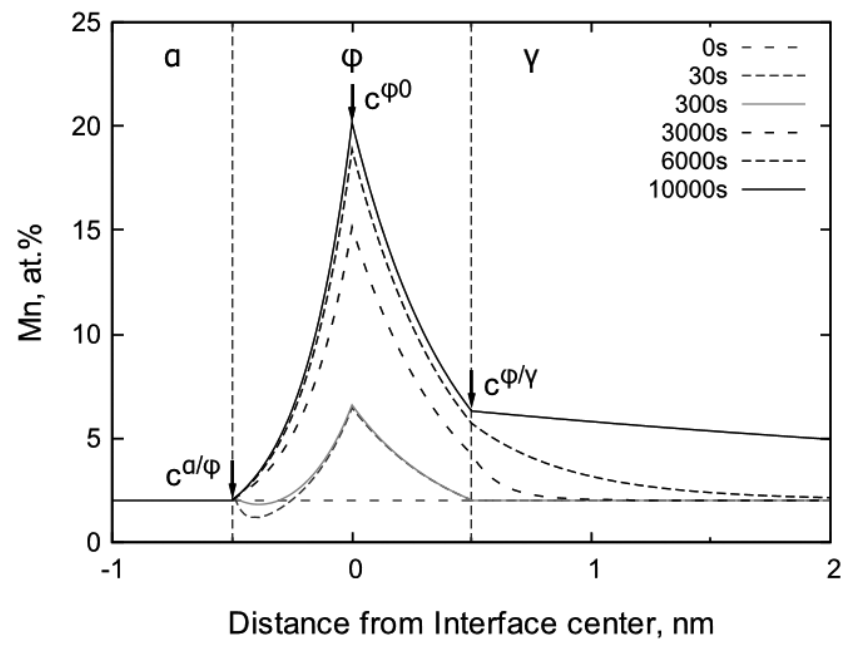

Fig. 6. Temporal evolution of Mn profiles across the migrating interface from $0 \mathrm{~s}$ to $10^{4} \mathrm{~s} . c_{M n}^{\alpha / \varphi}, c_{M n}^{\varphi 0}$ and $c_{M n}^{\varphi / \gamma}$ are the Mn concentrations at the $\alpha / \varphi$ boundary, the center of $\varphi$ and the $\varphi / \gamma$ boundary, respectively.

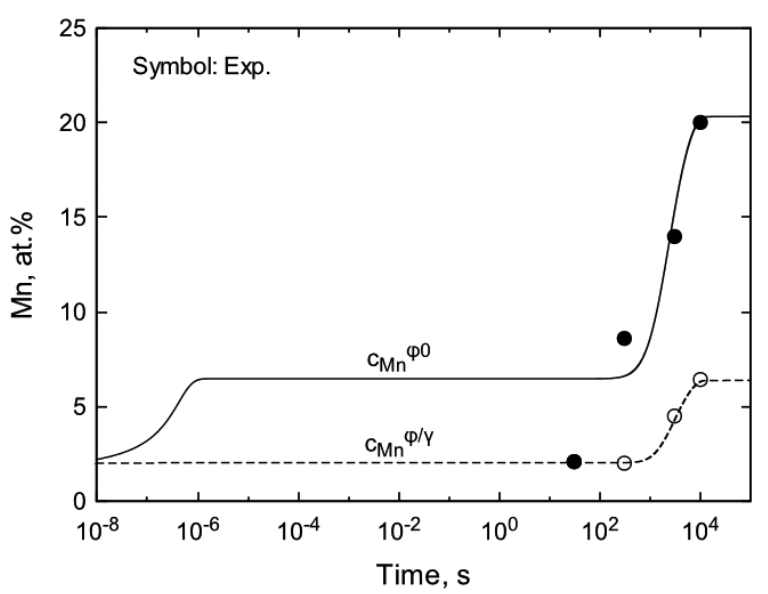

Fig. 7. Temporal change of the Mn concentrations at the center of $\varphi, c_{M n}^{\varphi 0}$ and at the $\varphi / \gamma$ boundary, $c_{M n}^{\varphi / \gamma}$. The solid circles are the experimental peak concentration of $\mathrm{Mn}$ in the interface region, $c_{M n}^{\varphi 0}$ and the open circles indicate the experimental Mn concentration at the boundary of the interface region and the austenite region, $c_{M n}^{\varphi / \gamma}$.

likely that the actual elapsed growth time of the ferrite grain observed in the experiment was longer than $30 \mathrm{~s}$.

At $300 \mathrm{~s}$, the Mn peak appears even for the experimental profile. The height of the experimental peak is 8.6 at.\%, while $c_{M n}^{\varphi 0}$ in the simulation is 6.6 at.\%. The concentrations at $\varphi / \gamma, c_{M n}^{\varphi / \gamma}$ for the experiment and the simulation remain the matrix concentration. The characteristics of the profiles for the experiment and the simulation are similar each other.

After $300 \mathrm{~s}, c_{M n}^{\varphi 0}$ and $c_{M n}^{\varphi / \gamma}$ increase with time. At 3000 $\mathrm{s}$ and $10000 \mathrm{~s}$, the calculated values of $c_{M n}^{\varphi 0}$ and $c_{M n}^{\varphi / \gamma}$ are almost the same as the values in the experiment.

\section{Discussion}

\subsection{The Transition from PE to NPLE}

The simulation results in Fig. 8 are discussed here. In Figs. 8(a) and 8(b) (for $30 \mathrm{~s}$ and $300 \mathrm{~s}$ ), $c_{M n}^{\varphi / \gamma}$ stays at the concentration of the $\gamma$ matrix, although the Mn peak in the interface region $\varphi$ can be seen. The Mn spike is not 
(a)

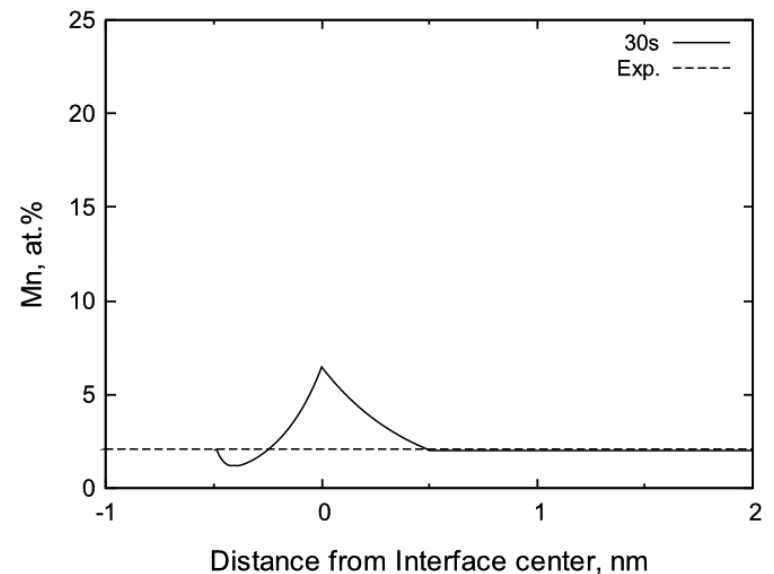

(b)

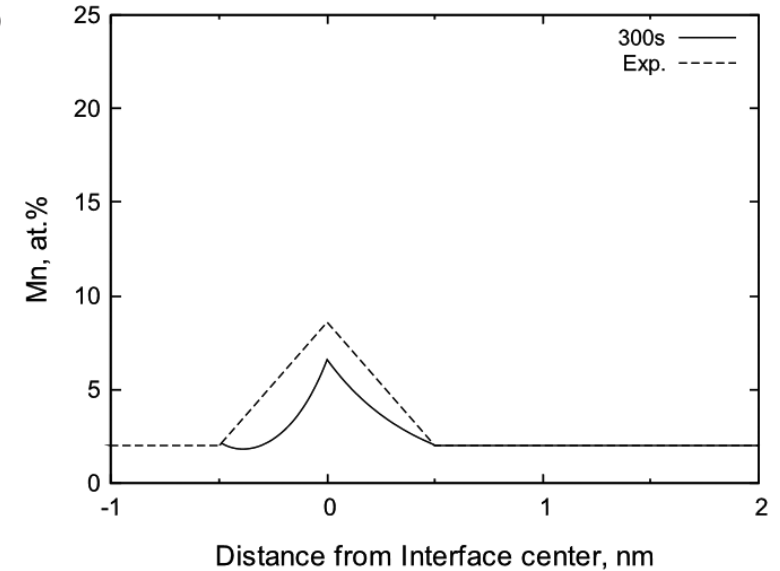

(c)

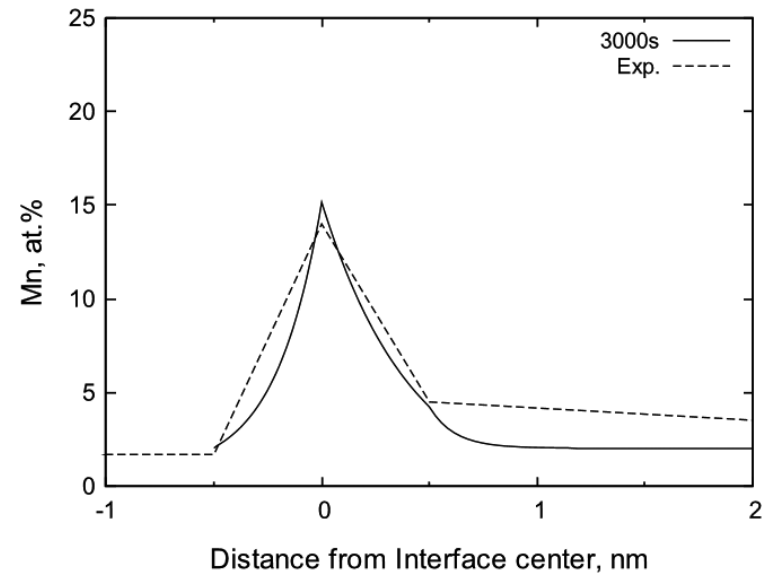

(d)

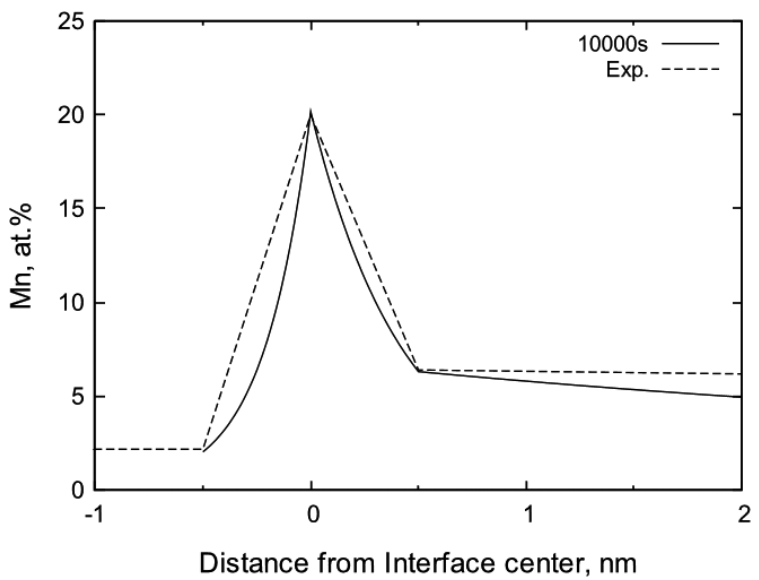

Fig. 8. A series of the Mn concentration profiles across the interface region $\varphi$ for the simulations and the TEM-EELS observation at (a) $30 \mathrm{~s}$, (b) $300 \mathrm{~s}$, (c) $3000 \mathrm{~s}$, and (d) $10000 \mathrm{~s}$. formed. This means that the condition of PE is maintained for $30 \mathrm{~s}$ and $300 \mathrm{~s}$. In Figs. 8(c) and 8(d) (for $3000 \mathrm{~s}$ and $10000 \mathrm{~s}), c_{M n}^{\varphi / \gamma}$ increases from the concentration of the $\gamma$ matrix according to the increase of the Mn peak in $\varphi$. This means that the Mn spike is formed in the front of the $\gamma$ side of $\varphi / \gamma$ after $3000 \mathrm{~s}$.

\subsection{The Path from PE to NPLE on the Isothermal Cross Section of the C-Mn Phase Diagram}

In order to obtain the path from PE to NPLE, the calculation of the $\mathrm{C}$ concentration change with time is necessary parallel to the calculation of the $\mathrm{Mn}$ concentration change. In the transition from PE to NPLE, the diffusion of C controls the migration of the $\alpha / \gamma$ interface. The temporal change of $\mathrm{C}$ concentration at the $\alpha / \gamma$ sharp interface during the austenite to ferrite transformation is calculated solving a diffusion equation with a quadratic concentration field approximation in front of the migrating interface. ${ }^{7,25)}$

From the mass balance between the growing phase $(\alpha)$ and the parent phase $(\gamma)$, the following expression is obtained for $\mathrm{C}$.

$$
S=\frac{L\left(c_{C}^{\gamma}-c_{C}^{b}\right)}{3\left(c_{C}^{b}-c_{C}^{\alpha}\right)} .
$$

where $S$ is the thickness of the $\alpha$ phase, $L$ is the diffusion length, $c_{C}^{\gamma}$ is the concentration of $\mathrm{C}$ at the interface in $\gamma, c_{C}^{b}$ is the $\mathrm{C}$ concentration in $\gamma, c_{C}^{\alpha}$ is the $\mathrm{C}$ concentration at the interface in $\alpha . c_{C}^{\alpha}$ is assumed to be zero, because it is small compared with $c_{C}^{b}$.

In the present case the flux balance equation may not be satisfied, because $c_{C}^{\gamma}$ is dependent on time. The expression for the temporal change of $c_{C}^{\gamma}$ is obtained from Eq. (16)

$$
c_{C}^{\gamma}(t)=c_{C}^{b}\left(1+\frac{3 S(t)}{L(t)}\right)
$$

Here, time $(t)$ is explicitly shown in the expression. In the present model, the curve fitted with the experimental result (Fig. 2(a)) was used for $S(t)$. The diffusion length $L(t)$ is assumed to be calculated by diffusion distance of $\mathrm{C}$ in $\gamma$ as follows.

$$
L(t)=K \sqrt{D_{C}^{\gamma} t}
$$

where $K$ is a constant, which is determined by the experimental condition that $c_{C}^{\gamma}(10000)=0.184 \mathrm{wt} . \%$. $D_{C}^{\gamma}$ was calculated using DICTRA ${ }^{24)}$ and the value of $3.24 \cdot 10^{-13}$ $\mathrm{m}^{2} / \mathrm{s}$ was obtained. For $K$ the value of 2.30 was calculated from the above condition.

Figure 9 shows the temporal change of $c_{C}^{\gamma}(t)$ together with that of Mn concentration at the $\varphi / \gamma \cdot c_{C}^{\gamma}(t)$ decreases rapidly from the value at PE, $0.55 \mathrm{wt} . \%$ at the early stage of the process, while $c_{M n}^{\varphi / \gamma}(t)$ gradually increases with time after 300 s. In Fig. 10, the path from PE to NPLE is shown with the thick black line on the isothermal $\mathrm{C}-\mathrm{Mn}$ section of $\mathrm{Fe}-\mathrm{C}-\mathrm{Mn}$ ternary phase diagram at $700^{\circ} \mathrm{C}$. The $\mathrm{C}$ and Mn concentrations at the $\varphi / \gamma$ change along the path. The partial local equilibrium condition is maintained between PE and NPLE. 


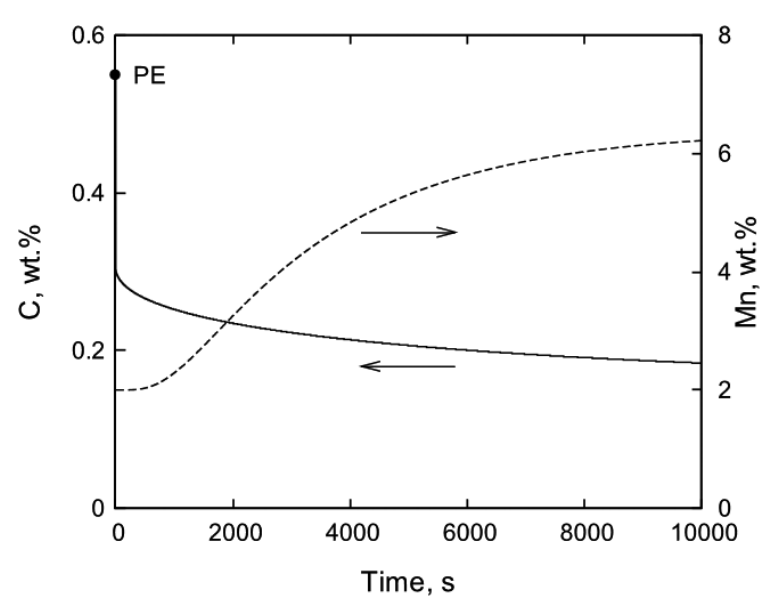

Fig. 9. Temporal change of the $\mathrm{C}$ concentration at the $\varphi / \gamma$ boundary. The change of Mn concentration at the $\varphi / \gamma$ boundary is plotted together.

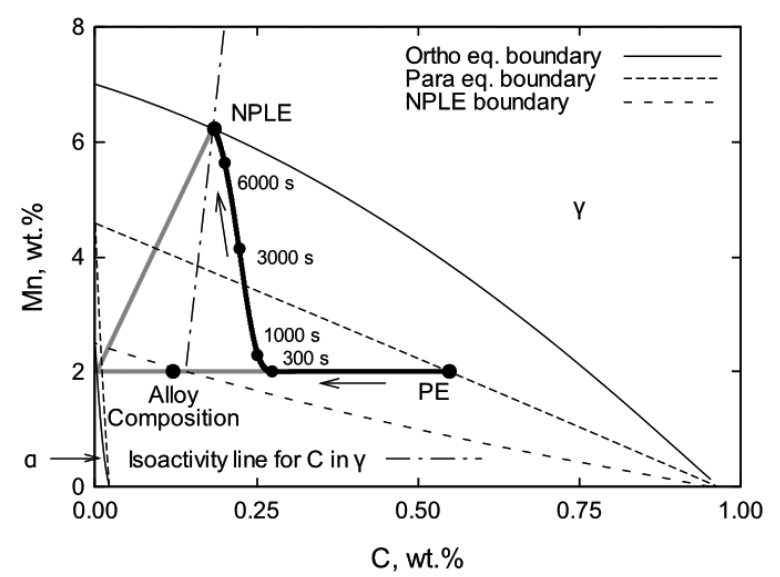

Fig. 10. The path from PE to NPLE during the simulation is plotted with thick black line on the Fe-rich side of $\mathrm{C}-\mathrm{Mn}$ section of $\mathrm{Fe}-\mathrm{C}-\mathrm{Mn}$ ternary phase diagram at $700^{\circ} \mathrm{C}$. The time elapsed during the simulation is indicated along the path. The boundaries of ortho-equilibrium, PE and NPLE are plotted on the diagram. The alloy, NPLE and PE compositions are shown by the large solid circles.

\subsection{Mn Spike}

Figure 11 shows the Mn profiles in the $\gamma$ matrix adjacent to the interface region $\varphi$. The penetration depth is considered to be the width of the Mn spike. The width of the Mn spike is defined as the distance from the $\varphi / \gamma$ boundary to the position in $\gamma$, where the Mn concentration becomes 1/e of $c_{M n}^{\varphi / \gamma}-c_{M n}^{b}$. Figure 12 shows the temporal change of the width of the Mn spike together with $D / v$. According to Coates $^{26)}$ and Hillert, ${ }^{2)}$ the width of the alloy spike could be described by $D / v$, a quantity that should be compared with the distance between atoms, $d(=0.26 \mathrm{~nm}$ for $\gamma$ iron $)$. The expression for the width of the spike, $D / v$ can be derived from Eq. (12). Figure 12 indicates that the width of the spike can be well described by $D / v$. Hillert ${ }^{2)}$ proposed that the local conditions at the interface should be full equilibrium only if $D / v>10 d$ and be close to $\mathrm{PE}$ if $D / v<0.4 d$. This criteria and Fig. 12 suggest that in the present simulation the condition at $\varphi / \gamma$ is close to the local equilibrium after $\sim 9500$ $\mathrm{s}$ and is close to PE before $\sim 2500 \mathrm{~s}$. This is consistent with Fig. 9. The time between $2500-9500 \mathrm{~s}$ is considered to be the transition period.

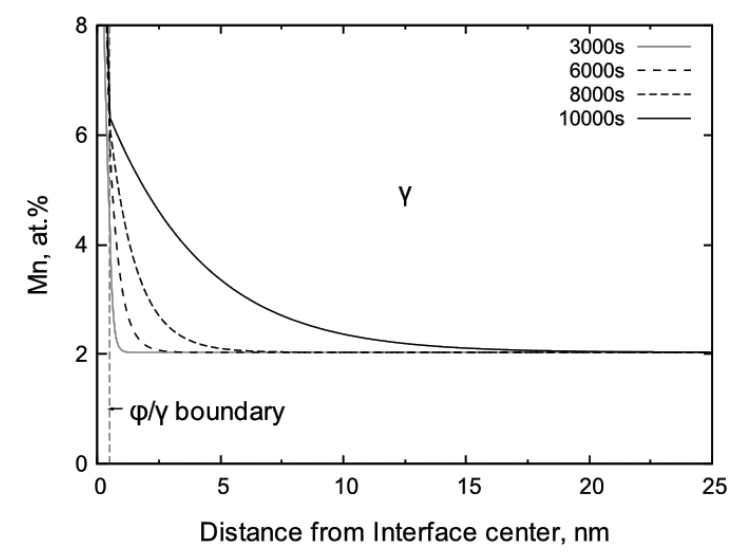

Fig. 11. Mn profiles in the $\gamma$ matrix adjacent to the interface region $\varphi$ from $3000 \mathrm{~s}$ to $10000 \mathrm{~s}$.

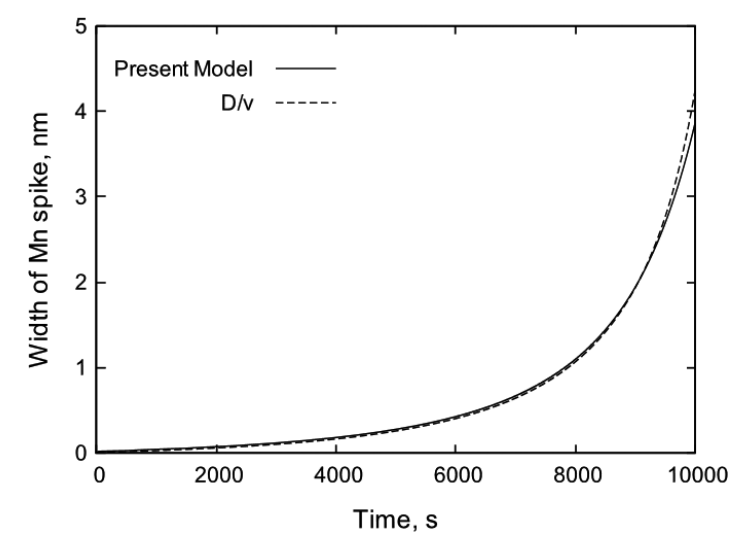

Fig. 12. Temporal change of the width of Mn spike (the solid line). The width is defined as the distance from the $\varphi / \gamma$ boundary to the position in $\gamma$, where the Mn concentration becomes $1 / e$ of $c_{M n}^{\varphi / \gamma}-c_{M n}^{b}$. The broken line indicates $D_{M n}^{\gamma} / v$, where $v$ is the interface migration velocity.

\subsection{Partitioning in the Process from PE to NPLE}

At $3000 \mathrm{~s}$ and $10000 \mathrm{~s}$ the experimental $\mathrm{Mn}$ profiles show much deeper diffusion penetration distance into the $\gamma$ matrix than the simulation ones (Figs. 8(c), 8(d)). In the simulations the depth of diffusion penetration is considered to be the Mn spike because the present simulations were performed under the non-partition or negligible-partition condition. This fact suggests that the partitioning of $\mathrm{Mn}$ occurred possibly at the early stage of the transformation in the experiment. ${ }^{19)}$

\subsection{Estimation of Effective Potential Depth $\boldsymbol{E}_{\mathbf{0}}$}

The potentials $E_{0}$ and $\Delta E$ are calculated from $f_{g b}$ and $f_{\gamma}$ using Eqs. (14) and (15). The activity coefficients $f_{g b}$ and $f_{\gamma}$ can be estimated using Hillert's grain boundary phase model $^{27)}$ and regarding grain boundaries as Liquid phase. The equilibrium between the liquid phase and the solid phases are calculated by parallel tangent law while the equilibrium between the ferrite and the austenite phases are calculated by common tangent law using Thermo-Calc. ${ }^{18)}$ The equilibrium concentrations at the interface at $700^{\circ} \mathrm{C} c_{\alpha}^{e}$, $c_{g b}^{e}$ and $c_{\gamma}^{e}$ are 1.24, 9.96 and 4.41 in at.\%, respectively. The following relations hold when $f_{\alpha}=1$.

$$
c_{\alpha}^{e}=f_{g b} c_{g b}^{e}=f_{\gamma} c_{\gamma}^{e}
$$


Using Eqs. (14), (15), (19), the values of $E_{0}$ and $\Delta E$ are obtained as follows.

$$
E_{0}=11.7 \mathrm{~kJ} / \mathrm{mol}, \Delta E=5.12 \mathrm{~kJ} / \mathrm{mol}
$$

These values are comparable with the ones used in the simulation. The method above to calculate the potential well is thought to be useful when experimental results are not available.

\subsection{Models of Diffusion Coefficient in the Interface Region}

In the present study, the diffusion coefficient of Mn inside the interface region, $D_{M n}^{\varphi}$ is assumed to be the geometric average of the diffusion coefficients of $\mathrm{Mn}$ in $\alpha$ and $\gamma . D_{M n}^{\varphi}$ is calculated using the following equation (Model 1).

$$
D_{M n}^{\varphi}=\sqrt{D_{M n}^{\alpha} \cdot D_{M n}^{\gamma}}
$$

where $D_{M n}^{\gamma}$ and $D_{M n}^{\alpha}$ are the diffusion coefficients of $\mathrm{Mn}$ in $\alpha$ and $\gamma$, respectively.

In the literatures, the other model to calculate the diffusion coefficient in the interface region has been proposed. ${ }^{7)}$ It is assumed to be the geometric average of the diffusion coefficients of $\mathrm{Mn}$ in $\alpha, \gamma$ and the grain boundary as follows.

$$
D_{M n}^{\varphi}=\sqrt[3]{D_{M n}^{\alpha} \cdot D_{M n}^{g b} \cdot D_{M n}^{\gamma}}
$$

where $D_{M n}^{g b}$ is the grain boundary diffusion coefficient. The grain boundaries for $\alpha$ or $\gamma$ are considered (Model 2 and Model 3). The grain boundary diffusion coefficients are calculated using the activation energy by factor 0.6 and the pre-factor of the $\alpha$ or the $\gamma$ diffusion coefficients. ${ }^{28)}$ Figure 13 shows the temporal evolution of the peak concentrations $c_{M n}^{\varphi 0}$ for these three models. The best agreement with the experiment is obtained for Model 1.

\subsection{Limitation of the Present Model}

In the present model, the velocity was calculated from the temporal change of the observed ferrite thickness shown in Fig. 2. As shown in the figure, the error bars are so large that the determination of the interfacial velocity is difficult especially in early stage of the transformation. Therefore, the present model has limitations for describing the PE stage

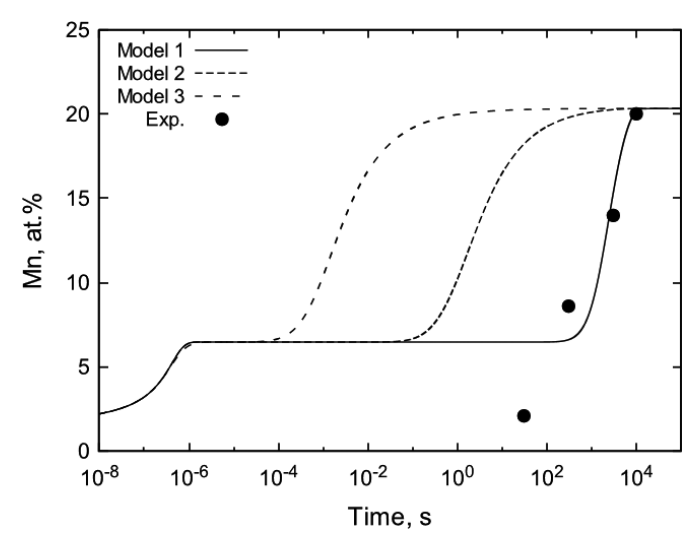

Fig. 13. Temporal change of the Mn concentration at the center of the interface region $\varphi, c_{M n}^{\varphi 0}$ for three models used to calculate the diffusion coefficient of $\mathrm{Mn}$ in the interface region. The best agreement with the experiment is obtained for Model 1. and the early stage of the transition process from PE to NPLE. In future improved models, the mechanism of interfacial velocity calculation must be included. Furthermore, to describe the $\mathrm{PE}$ stage, where diffusion of $\mathrm{C}$ plays an important role, the diffusion of $\mathrm{C}$ and $\mathrm{Mn}$ must be calculated simultaneously. ${ }^{8)}$

\section{Conclusions}

A non-steady state (time-dependent) model describing the transition from the para-equilibrium to the local equilibrium of austenite-to-ferrite transformation in $\mathrm{Fe}-\mathrm{C}-\mathrm{X}$ alloys under the negligible- or non- partition condition has been developed. The temporal evolution of the Mn profiles can be obtained using the present model. The model was applied to the $\mathrm{Fe}-0.12 \% \mathrm{C}-2 \% \mathrm{Mn}$ alloy. The migration velocity of the $\alpha / \gamma$ interface must be given in the model. The observed interface velocity was used here. In the simulation, the depth of the triangular potential in the interface region was used as a fitting parameter for the experimental results. The Mn profiles obtained by the simulations agreed very well with the observed ones by STEM-EELS. Combining a simple C diffusion model with the present non-steady state calculation for $\mathrm{Mn}$, the transition path from PE to NPLE on the C-Mn isothermal section of $\mathrm{Fe}-\mathrm{C}-\mathrm{Mn}$ alloys was presented.

\section{REFERENCES}

1) D. E. Coates: Metall. Trans., 4 (1973), 2313

2) M. Hillert: Scr. Mater., 46 (2002), 447.

3) A. Hultgren: Trans. Am. Soc. Met., 39 (1947), 915

4) J. Odqvist, M. Hillert and J. Ågren: Acta Mater., 50 (2002), 3211.

5) G. R. Purdy and Y. J. M. Brechet: Acta Mater., 43 (1995), 3763.

6) H. Chen, K. Zhu, L. Zhao and S. van der Zwaag: Acta Mater., 61 (2013), 5458.

7) H. Chen and S. van der Zwaag: Acta Mater., 72 (2014), 1.

8) M. Enomoto: Acta Mater., 47 (1999), 3533.

9) H. Guo and M. Enomoto: Metall. Mater. Trans. A, 38 (2007), 1152.

10) H. Chen, Z. Yang, C. Zhang, K. Zhu and S. van der Zwaag: Acta Mater., 104 (2016), 62.

11) S. J. Clark, Y. Lan, A. Rahnama, V. Janik and S. Sridhar: ISIJ Int., 59 (2019), 573.

12) H. Dong, H. Chen, W. Wang, Y. Zhang, G. Miyamoto, T. Furuhara, C. Zhang, Z. Yang and S. van der Zwaag: Acta Mater., 158 (2018), 167.

13) T. Murakami, H. Hatano, G. Miyamoto and T. Furuhara: ISIJ Int., 52 (2012), 616.

14) J. W. Cahn: Acta Metall., 10 (1962), 789.

15) M. Hillert and B. Sundman: Acta Metall., 24 (1976), 731.

16) M. Hillert, J. Odqvist and J. Ågren: Scr. Mater., 45 (2001), 221.

17) G. R. Speich, V. A. Demarest and R. L. Miller: Metall. Trans. A, 12 (1981), 1419.

18) N. Nakada, K. Mizutani, T. Tsuchiyama and S. Takaki: Acta Mater., 65 (2014), 251.

19) T. Amino, G. Shigesato, T. Nozaki and M. Azuma: Proc. 5th Int Symp. on Steel Science, ISIJ, Tokyo, (2017), 55.

20) G. Shigesato, T. Fujishiro and T. Hara: Mater. Sci. Eng. A, 556 (2012), 358.

21) T. Nishizawa: Thermodynamics of Microstructures, ASM International, Materials Park, OH, (2008), 77.

22) H. Takami and T. Kawamura: Numerical Solutions of Partial Differential Equations by the Finite Difference Method, University of Tokyo Press, Tokyo, (1994), 24 (in Japanese).

23) W. H. Press, B. P. Flannery, S. A. Teukolsky and W. T. Vetterling: Numerical Recipes, Cambridge University Press, Cambridge, (1986), 637.

24) Thermo-Calc Software, http://www.thermocalc.com/, (accessed 201903-26).

25) H. Chen and S. van der Zwagg: J. Mater. Sci., 46 (2011), 1328

26) D. E. Coates: Metall. Trans., 3 (1972), 1203.

27) M. Hillert: Phase Equilibria, Phase Diagrams and Phase Transformations, Cambridge University Press, Cambridge, (1998), 392.

28) A. P. Sutton and R. W. Balluffi: Interfaces in Crystalline Materials, Clarendon Press, Oxford, (1995), 476. 